\title{
Immunomodulatory strategies prevent the development of autoimmune emphysema
}

\author{
Masayuki Hanaoka', Mark R Nicolls², Andrew P Fontenot', Donatas Kraskauskas³ ${ }^{3}$ Douglas G Mack', \\ Adelheid Kratzer', Jonas Salys ${ }^{1}$, Vita Kraskauskiene ${ }^{3}$, Nana Burns ${ }^{1}$, Norbert F Voelkel ${ }^{3}$, \\ Laimute Taraseviciene-Stewart $^{1 *}$
}

\begin{abstract}
Background: The presence of anti-endothelial cell antibodies and pathogenic T cells may reflect an autoimmune component in the pathogenesis of emphysema. Whether immune modulatory strategies can protect against the development of emphysema is not known.

Methods: Sprague Dawley rats were immunized with human umbilical vein endothelial cells (HUVEC) to induce autoimmune emphysema and treated with intrathymic HUVEC-injection and pristane. Measurements of alveolar airspace enlargement, cytokine levels, immuno histochemical, western blot analysis, and T cell repertoire of the lung tissue were performed.

Results: The immunomodulatory strategies protected lungs against cell death as demonstrated by reduced numbers of TUNEL and active caspase-3 positive cells and reduced levels of active caspase-3, when compared with lungs from HUVEC-immunized rats. Immunomodulatory strategies also suppressed anti-endothelial antibody production and preserved CNTF, IL-1alpha and VEGF levels. The immune deviation effects of the intrathymic HUVEC-injection were associated with an expansion of CD4+CD25+Foxp3+ regulatory $T$ cells. Pristane treatment decreased the proportion of $T$ cells expressing receptor beta-chain, $V \beta 16.1$ in the lung tissue.

Conclusions: Our data demonstrate that interventions classically employed to induce central $\mathrm{T}$ cell tolerance (thymic inoculation of antigen) or to activate innate immune responses (pristane treatment) can prevent the development of autoimmune emphysema.
\end{abstract}

\section{Background}

It is now increasingly recognized that chronic obstructive pulmonary disease (COPD)/emphysema presents clinically as a syndrome with pulmonary and extrapulmonary manifestations $[1,2]$. Chronic inflammatory mechanisms are being discussed as important factors which either trigger or maintain this chronic disorder [3-5]. Both lymphocytic infiltration of the bronchial mucosa and lung parenchyma [6,7] as well as lymph follicles in close proximity to bronchioles have been described [8]. It has been hypothesized that there may be an autoimmune component to the chronic progressive lung tissue destruction which can persist after

\footnotetext{
* Correspondence: Laima.Taraseviciene@ucdenver.edu

'Department of Medicine, University of Colorado Denver, Aurora, CO, 80045, USA

Full list of author information is available at the end of the article
}

smoking cessation [9-12]. Recently Lee and co-workers demonstrated anti-elastin autoantibodies in patients with tobacco smoking-induced emphysema [13]. Autoimmune diseases result from the propagation of self-reactive $\mathrm{T}$ and $\mathrm{B}$ lymphocytes that recognize selfantigens and mediate tissue destruction.

Our group has described a rat autoimmune emphysema model which is characterized by anti-endothelial cell antibodies (AECA) and pathologic T lymphocytes, since both plasma as well as splenocytes have been shown to trigger emphysema after passive transfer into healthy naïve rodents [14], suggesting that the preexisting $\mathrm{T}$ cell repertoire in the lung is not sufficient to respond to xenogeneic antigens. We reasoned that this autoimmune model of emphysema, which is based on immunization of rats with xenogeneic human umbilical vein endothelial cells (HUVEC), can be utilized to 
demonstrate the phenomenon of immune tolerance, because autoimmunity is due to the breakdown of central and/or peripheral tolerance. If so, then demonstration of protection against emphysematous lung destruction by immune modulation would provide distinct support for the role of an autoimmune contribution to lung destruction in this rodent model [14]. This concept has been repeatedly demonstrated in the NOD (non-obese diabetic) model of autoimmune diabetes in which multiple interventions in young mice skew the immune response and prevent disease $[15,16]$.

In the present study, we utilize interventions classically employed in experimental autoimmune models to induce central $\mathrm{T}$ cell tolerance by thymic inoculation of antigen [17] and to activate innate immune responses with pristane [18]. The acquisition of $\mathrm{T}$ cell tolerance to self occurs primarily within the thymus $[19,20]$ and can be achieved through 3 not mutually exclusive mechanisms: clonal deletion, clonal anergy and specific suppression. Intrathymic tolerance induction by inoculation of donor bone marrow cells, spleen cells and other type of cells has been successfully used to prolong the survival of cardiac, skin and islet allografts in adult rodents [20-23]. Here we expand previous studies by utilizing an intrathymic inoculation approach in our animal model of autoimmune emphysema. The hydrocarbon oil adjuvant pristane has been shown to induce a persistent decrease in phytohemagglutinin responsiveness, consistent with a block of polyclonal $\mathrm{T}$ cell proliferation [18]. By demonstrating that early immunomodulation prevents experimental emphysema in pathogen-free animals, the concept that autoimmune injury may contribute to clinical emphysema is further bolstered.

\section{Methods}

\section{Experimental protocols}

The experimental protocol was approved by the Animal Care and Use Committee of the University of Colorado Denver. In our study we used 6 week old male SpragueDawley rats (body weight $200 \mathrm{~g}$ ). Animals were divided into four groups ( $\mathrm{n}=6$ per group): (1) control group (IP injection of phosphate buffered saline (PBS)), (2) HUVEC (H group) alone $\left(10 \times 10^{6}\right.$ cells, single IP injection[14]), (3) intrathymic injection of HUVEC (H-T-H group) (200,000 cells) 2 weeks prior to immunization with HUVEC; (4) pristane (H-P group) (200 $\mu$ l, single IP injection) 3 days prior to immunization with HUVEC. Pristane (2,6,10,14-tetramethyl-pentadecane) was purchased from Sigma-Aldrich Co. (St. Louis, MO). While in our previous study we performed immunization with adjuvant Titermax Gold, later we found out that HUVEC itself are strong immunogens and no adjuvant is needed for antibody production. In the present study we omitted this step of adjuvant administration.
Primary cultures of HUVEC were isolated in our laboratory following procedures described by Bruneel et al [24].

\section{Intrathymic injections}

Intrathymic injections were performed under anesthesia induced with a combination of ketamine $(100 \mathrm{mg} / \mathrm{kg}) /$ xylazine $(15 \mathrm{mg} / \mathrm{kg})$. The thymus gland was exposed through a small incision above the sternum and 200,000 HUVEC cells in $100 \mu \mathrm{l}$ of PBS were injected into thymic lobes $(50 \mu \mathrm{l} /$ per lobe). The control group received only PBS injections. The incision was closed with a 4-0 nylon suture. The injections were performed into 6 week old rats (body weight 200 g) 2 weeks prior to immunization with HUVEC. Indeed, these are very young rats and prior literature [25-27] supports intrathymic injection at 6 weeks.

\section{Tissue processing}

All the animals were sacrificed 3 weeks after the IP HUVEC treatment or PBS injection [14]. Lung tissue was obtained by opening the chest wall. Lungs were ligated. The right lung was inflated with $0.5 \%$ low melting agarose at a constant pressure of $25 \mathrm{cmH}_{2} \mathrm{O}$ and fixed in $10 \%$ formalin for $48 \mathrm{hr}$. Lung sections were embedded in paraffin using standard techniques. The upper and lower lobes of the left lung were snap-frozen for protein extraction.

\section{Lung morphology}

The $5 \mu \mathrm{m}$ thick sections were stained with hematoxylin and eosin. Ten images per slide were acquired at x200 magnification with an AxioCam Color Camera using an Axioskop 2 Microscope (Carl Zeiss MicroImaging Inc., Thornwood, NY). The degree of emphysema was quantified by measuring alveolar airspaces (in pixels per square micrometer [14]) and mean linear intercept (MLI; [28]). The CD4+ cell accumulation areas were measured in pixels per square micrometers. A macro was created to automate the segmentation, processing, and quantification steps. For each animal, 10 fields at a magnification of $\mathrm{x} 100$ were captured in a blinded fashion using Carl Zeiss KS300 Imaging System software.

\section{Cell death assessment and western blot (WB) analysis}

Terminal deoxynucleotidyl transferase-mediated dUTP nick end-labeling (TUNEL) was performed with TACS 2 TdT DAB kit (Trevigen, Gaithersburg, MD) following manufacturer's protocol. The ratio of TUNEL-positive cells to total cells (apoptotic index) was measured assessing more than 3,000 parenchymal cells of each lung sample in each group [29].

Active caspase- 3 was assessed using a rabbit polyclonal antibody to cleaved caspase-3 (Cell Signaling 
Technology Inc., Beverly, MA). Immunohistochemical staining was performed using VECTASTAIN ABC kit (Vector Laboratories Inc., Burlingame, CA).

WB analysis was performed as previously described [14]. Anti-CD19 antibodies were from Santa Cruz Biotechnology Inc., Santa Cruz, CA, and anti-CD68 from Serotec Ltd, Oxford, UK.

\section{Cytokine assay}

Cytokine levels in whole lung tissue homogenates were determined using Quantibody Rat-1 Array (RayBiotech, Inc, Norcross, GA) that allows measurement of the following cytokines: CINC-2 (CXCL3/GROgamma/DCIP1), CINC-3 (CXCL3/GRObeta/MIP-2), Fractalkine (CX3CL1), CNTF, GM-CSF, IL-1 $\alpha$, IL-4, LIX (CXCL5), $\beta$-NGF and VEGF.

\section{Lung and spleen $\mathrm{T}$ cell isolation}

Cells were isolated as described by Finotto et al., [30]. Negative selection of CD4+ T cells was performed using Rat CD4+ T Cell Enrichment Column from R\&D Systems, Inc. (Minneapolis, MN). The aliquot of the sorted cell preparation $(100 \mu \mathrm{l})$ was cytospinned, stained with Wright stain and counted. The CD4+ T cells isolated from the lungs were identified by flow cytometry. We also did the CD3 staining to confirm that the CD4+ cells were $\mathrm{T}$ cells and not, for example, strange macrophages.

\section{Lung CD4+ $T$ cell measurements}

Lung sections were stained with an anti-CD4 antibody and CD4+ cells were counted in 5 different lung fields. The CD4+ cell accumulation areas were measured in pixels per square micrometers. A macro was created to automate the segmentation, processing, and quantification steps using a Carl Zeiss KS300 Imaging System software. A CD4+ T cell count was also performed on cells isolated from the lungs. A CD4+ T cell count was also performed on cells isolated from the lungs by flow cytometry. Both methods gave comparable results.

\section{Flow cytometry}

Isolated CD4+ T cells were washed in PBS supplemented with $1 \%$ bovine serum albumin (BSA) (FACS buffer) and surface stained with $\mathrm{mAbs}$ directed against $\mathrm{CD} 3$ (FITC, Invitrogen) and CD4 (APC, Invitrogen or BD) along with one of the following $\mathrm{mAbs}$ to TCR $\mathrm{V}$ beta $(8.5,10$, or 16.1 (AbD Serotec) or CD25 (PE, Biolegend) for $30 \mathrm{~min}$ at $4^{\circ} \mathrm{C}$ as described previously [31]. Cells were washed in FACS buffer and stained with secondary antibodies to each TCR V beta (R-PE, Invitrogen). For intranuclear staining with anti-Foxp3 (PE; eBiosciences), surface stained cells were washed twice, fixed and permeabilized prior to staining with anti-Foxp3. Cells were washed and resuspended in $1 \%$ formaldehyde. The number of events collected ranged between 1 and 3 million. Samples were acquired on a FACSCalibur equipped with $488 \mathrm{~nm}$ argon and $633 \mathrm{~nm}$ red-diode laser for four color detection. Analysis was performed using FlowJo (Treestar) software. Lymphocytes were gated using forward scatter versus side scatter.

\section{Statistical analysis}

All data are expressed as mean \pm SEM. Statistical analysis was performed with the Statview software package (SAS Institute Inc., Cary, NC). The comparison between two groups was performed by employing the Student's unpaired $t$ test for independent samples. The values obtained in the different groups of rats were compared using one-way ANOVA. Statistical difference was accepted at $\mathrm{p}<0.05$.

\section{Results}

Immunomodulation strategies protect against airspace enlargement

As previously reported [14], when compared to lungs from PBS-injected control animals (Figure 1A), histological examination of lung tissue samples demonstrated diffusely enlarged airspaces in HUVEC-immunized rats (Figure 1B). In contrast, there was no alveolar space enlargement in the lungs from animals treated with either intrathymic HUVEC-injection (Figure 1C) or pristane (Figure 1D) in association with intraperitoneal HUVEC injection. The quantitative data of alveolar airspace surface area and mean linear intercept (MLI) from all treatment groups are shown in Figure $1 \mathrm{E}$ and Figure 1F. The sections evaluated were selected in a random fashion.

\section{Immunomodulation strategies protect against lung cell apoptosis}

Both of the immunomodulatory treatments protected lungs against cell death as shown by the reduced numbers of TUNEL positive cells. Figure 2A shows PBS-treated control rat lung. When compared to lungs from HUVEC-immunized rats (Figure 2B), intrathymic injection of HUVEC followed by HUVEC immunization (Figure 2C) and pristane treatment (Figure 2D) significantly reduced TUNEL-positive cells in the rat lungs. The quantitative analysis of TUNEL positive cells is shown as an apoptotic index (Figure 2E).

\section{Immunomodulation strategies reduce lung inflammation and prevent caspase-3 activation}

Earlier we have reported [14] that during the first week of the immunization there was no difference in the numbers of CD19 or CD68 cells in immunized or control rat lungs. However, the accumulation of $\mathrm{B}$ cells 


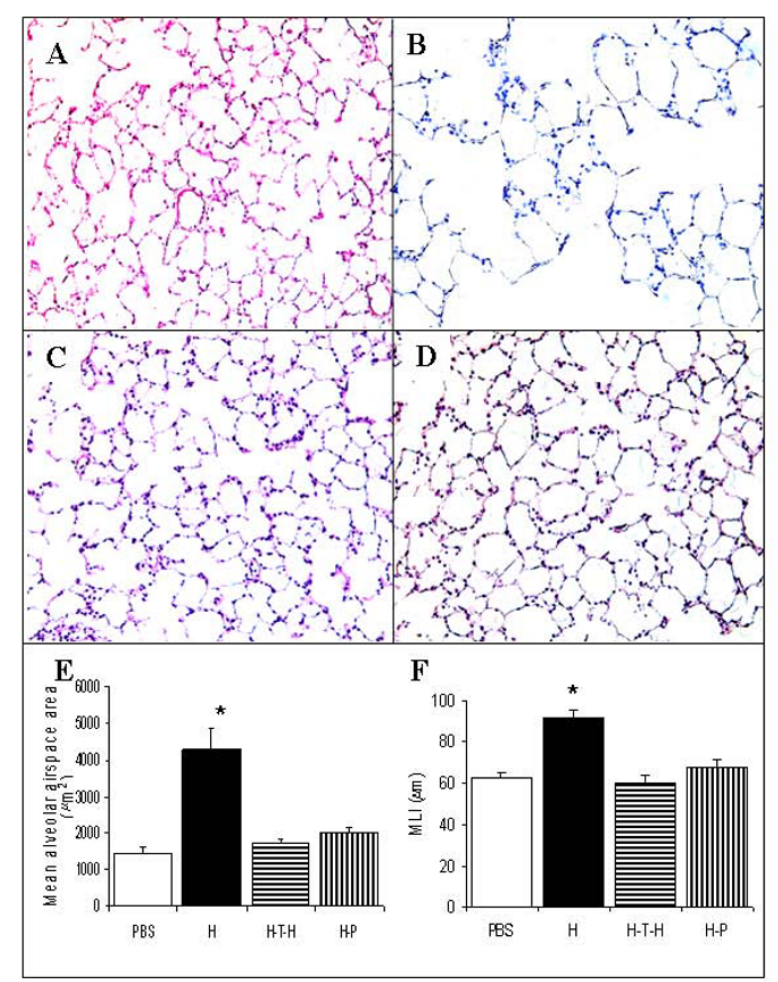

Figure 1 Morphology of rat lungs and alveolar airspace measurements. Hematoxylin and eosin (H\&E) staining of rat lungs ( $n=6$ in each group): (A) PBS-treated control (PBS); (B) HUVECimmunized (H); (C) Intrathymically HUVEC-injected and HUVECimmunized $(\mathrm{H}-\mathrm{T}-\mathrm{H})$; (D) Pristane-treated 3 days prior to HUVECimmunization (H-P). (E) Quantitation of mean alveolar airspace area (micrometer ${ }^{2}$ ); (F) Mean linear intercept (MLI). Magnification x200.* $p$ $<0.05$ compared to control and each of tolerized rat groups.

(CD19+; Figure 3A) was observed in rat lungs at 1.5-3 weeks after HUVEC-injection. The number of these cells in the lung tissue was attenuated by intrathymic HUVEC-injection (Figure 3B) and pristane treatment (Figure 3C). The accumulation of macrophages as shown by immunohistochemistry for CD68 in HUVECimmunized rat lungs (Figure 3D) was reduced by intrathymic HUVEC-injection (Figure 3E) and pristanetreatment (Figure 3F).

Because TUNEL staining detects both apoptotic and necrotic cells, to identify apoptotic events in the lung tissue, we performed immunohistochemistry (IHC) for active caspase-3 (Figure 3G - HUVEC-immunized; Figure $3 \mathrm{H}$ - intrathymic HUVEC-injection and Figure 3I pristane-treated rat lungs). The number of active caspase-3 positive cells was markedly reduced by both of the tolerizing strategies.

We confirmed the IHC findings by performing quantitative Western blot (WB) analysis of whole lung tissue extracts. The levels of activated caspase-3, CD68 and

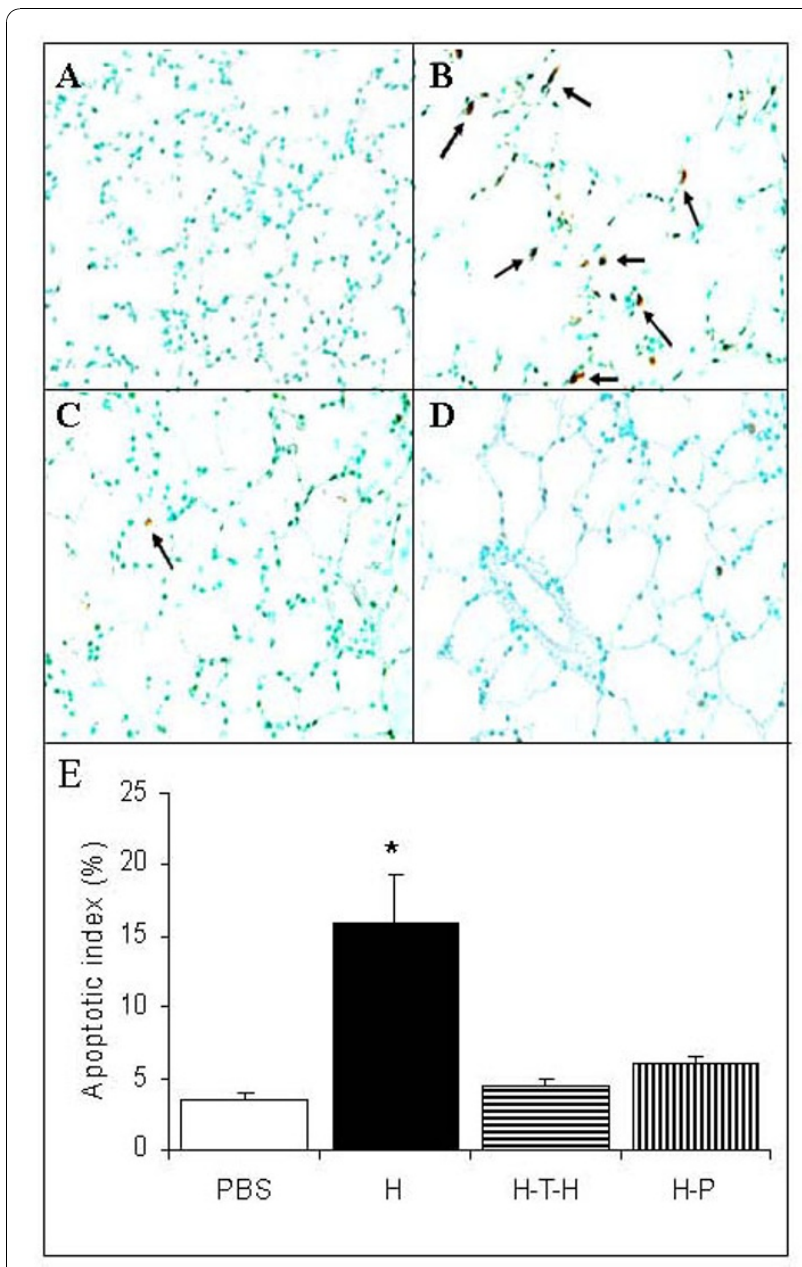

Figure 2 Apoptosis in rat lungs and apoptotic index. Cell death in rat lungs $(n=6)$ assessed by TUNEL staining. (A) PBS-treated control; (B) HUVEC-immunized; (C) Intrathymically HUVEC-injected and HUVEC-immunized; (D) Pristane-treated 3 days prior to HUVECimmunization. (E) The apoptotic index (\%) was calculated as the ratio of TUNEL-positive cells to total lung cells. Magnification $\times 400$. ${ }^{*} p<0.05$ compared to control and each of tolerized rat groups.

CD19 were significantly higher in the HUVEC-immunized rats compared to the PBS-treated controls (Figure 3J). The induction of central tolerance with intrathymic HUVEC-injection and the immunomodulatory strategy using pristane attenuated the expression of caspase- 3 as well as the expression of CD68 and CD19.

\section{Immunomodulation and T cells}

Usually, in normal rat lung, very few $\mathrm{CD} 4+\mathrm{T}$ cells can be found (Figure 4A). Conversely, in HUVEC-injected rat lungs there is a significant accumulation of $\mathrm{CD} 4+\mathrm{T}$ cells (Figure 4B). Tolerizing strategies such as thymic injection of antigen (Figure $4 \mathrm{C}$ ) and pristane treatment (Figure 4D) reduced the CD4+ cell numbers to the normal level. We have quantified the accumulation of CD4 


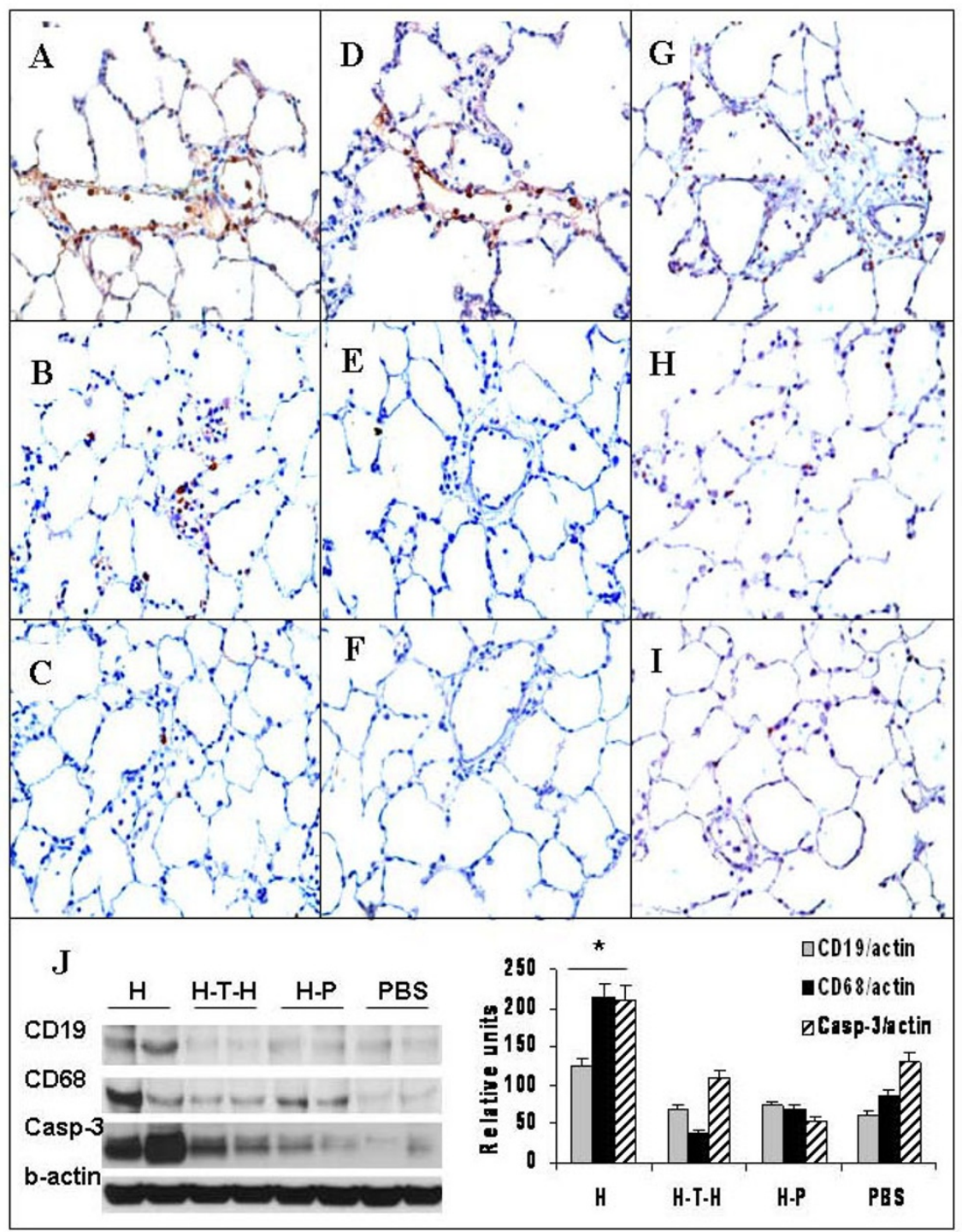

Figure 3 Immunostaining for B cells (CD19), macrophages (CD68) and active caspase-3 and western blot (WB) analysis. CD19 (A-C); CD68 (D-F) and active caspase-3 (G-I) of HUVEC-immunized (A, D, G); intrathymically HUVEC-injected and HUVEC-immunized (B, E, F); and pristane-treated 3 days prior to HUVEC-immunization (C, F, I) rat lung sections. Magnification x400. (J) Quantitative WB analysis of rat lung tissue homogenates for CD19, CD68 and active caspase-3. ${ }^{*} p<0.01$ compared to control or immune tolerized rats.

+ cells on IHC slides using the Carl Zeiss KS300 Imaging System software. As shown in Figure 4E, there was almost a 3-fold increase in the CD4+ T cell numbers in the HUVEC-immunized rat lungs when compared to untreated controls or rats treated with thymic injection of antigen or with pristane lungs. CD4+ cells from 4 rats per group were counted.

\section{Pristane treatment suppresses specific T cell receptor (TCR) expression}

Pristane treatment decreased numbers of CD4+ T cells in the lung tissue. Here we have tested whether pristane treatment affected $\mathrm{T}$ cell receptor expression in the lung by testing the expression of TCR V $\beta 16.1$, TCR V $\beta 8.5$ and TCR V $\beta 10$ in the treated and untreated HUVECimmunized rat lungs. There was a higher expression of TCR V $\beta 8.5$ in lungs and spleens from pristane-treated and HUVEC-immunized rats when compared to normal controls. The expression of TCR V $\beta 10$ was similar in all 3 groups. While there was no significant difference in TCR V $\beta$ expression on CD4+ T cells obtained from lung and spleen of normal controls and HUVEC-immunized animals ( $\mathrm{n}=4$ rats per group), there was a significantly decreased expression of $\mathrm{V} \beta 16.1$ in both 


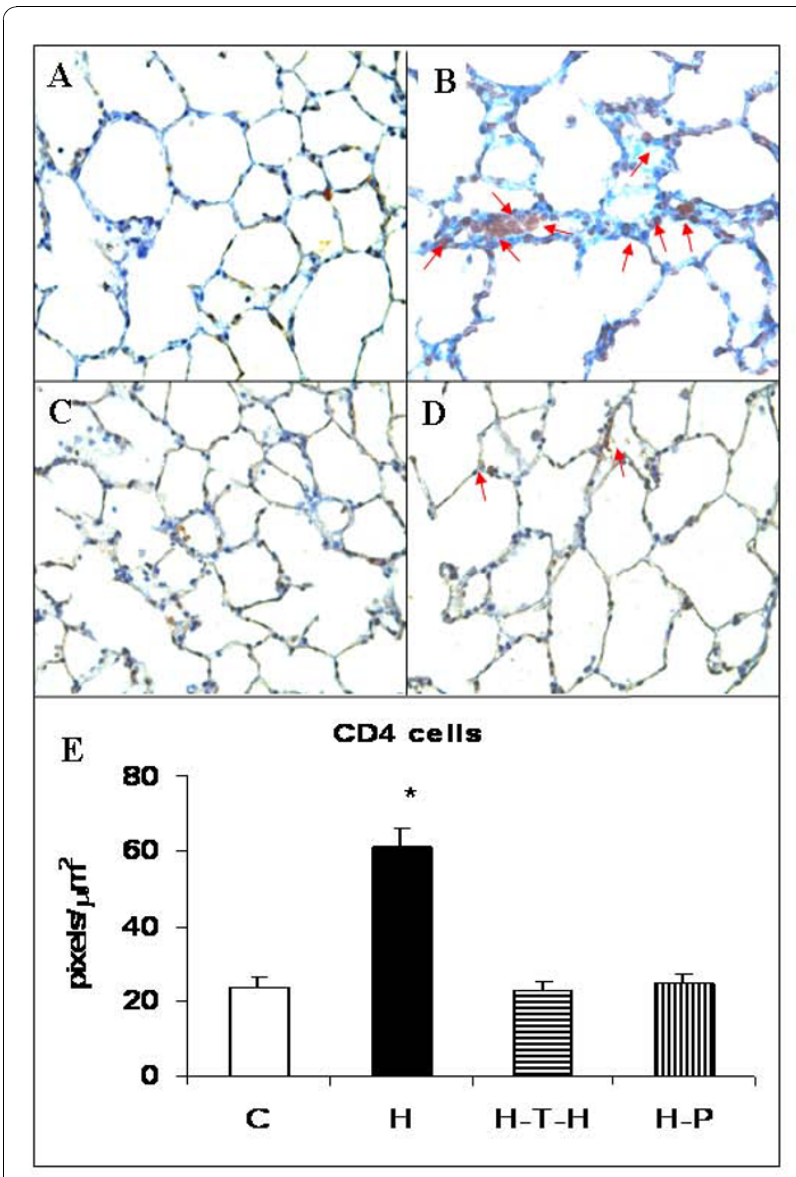

Figure 4 Immunostaining for CD4+ $\mathbf{T}$ cells. Immunohistochemistry for CD4+ T cells in PBS-treated (A); HUVECimmunized (B); intrathymically HUVEC-injected and HUVECimmunized $(\mathbf{C})$; and pristane-treated 3 days prior to HUVECimmunization (D) rat lung sections. Magnification x400. (E) The CD4 + cell accumulation areas were measured in pixels per square micrometers. A macro was created to automate the segmentation, processing, and quantification steps. For each animal $(n=4), 10$ fields at a magnification of $\times 100$ were captured in a blinded fashion using Carl Zeiss KS300 Imaging System software. * $p \leq 0.002$ when compared to immune tolerized rats.

lungs $(\mathrm{p} \leq 0.008)$ and spleens $(\mathrm{p} \leq 0.003)$ of pristane treated rats compared to rats receiving only HUVEC (Table 1). Similar findings were seen with the intrathymic injection of HUVEC prior to IP immunization in lungs and spleen $(9.03 \pm 0.94 \%$ and $8.83 \pm 0.47 \%$ respectively) when compared to HUVEC-immunized rats $(11.5 \pm 0.88 \%$ and $12.1 \pm 0.4 \% ; \mathrm{p} \leq 0.03$ and $\mathrm{p} \leq 0.007)$. Since HUVEC-immunized rats have 3-times more of such $\mathrm{CD} 4+\mathrm{T}$ cells in the lungs, these findings might suggest that pristane and intrathymic injection of HUVEC targeted a specific CD4+ T cell subset and raise the possibility that CD4+ T cells expressing TCR V $\beta 16.1$ may represent the pathogenic $\mathrm{T}$ cell population.
Intrathymic injection of antigen prior to immunization induces expansion of regulatory $\mathrm{T}$ (Treg) cells

Recent findings indicate that targeted deletion of Treg cells causes spontaneous autoimmune disease in mice, whereas augmentation of Treg-cell function can prevent the development of - or alleviate - variants of experimental autoimmune encephalomyelitis [32]. As shown in Table 2 levels of Foxp3+/CD25+/CD4+ Treg cells in the normal control rat lungs and spleen are extremely low. However, intrathymic injection of antigen prior to IP immunization resulted in a significantly increased percentage of Foxp3-expressing CD4+CD25+ Treg cells when compared with HUVEC-immunized rats, suggesting that the induction of central tolerance results in an expansion of naturally-occurring Treg cells $(n=4$ rats per group).

\section{Pristane treatment ameliorated the increase in lung tissue} cytokine levels

To examine whether induction of immune deviation by pristane can affect lung cytokine levels in lung tissue homogenates, we used Quantibody Rat-1 Array that allows measurements of the following cytokines: cytokine induced neutrophil chemoattractant-2 (CINC-2 also known as CXCL3/GROgamma/DCIP-1); cytokine induced neutrophil chemoattractant-3 (CINC-3 also known as CXCL3/GRObeta/MIP-2); GranulocyteMacrophage Colony Stimulating Factor (GM-CSF); lipopolysaccharide-induced CXC chemokine (LIX also known as CXCL5); interleukin-4 (IL-4); interleukin 1alpha (IL-1 $\alpha$ ); ciliary neurotrophic factor (CNTF); Fractalkine (CX3CL1), nerve growth factor beta ( $\beta$-NGF) and vascular endothelial growth factor (VEGF). HUVEC-immunization resulted in a greater than 40-fold increase in IL-1 $\alpha$, and a decrease in vascular endothelial growth factor (VEGF) and CNTF, levels (Figure 5A), whereas pretreatment with pristane preserved the normal VEGF and CNTF levels in the lung and significantly decreased IL-1 $\alpha$, levels in the HUVEC-immunized animals.

\section{Immunomodulation strategies protect abolished AECA production}

Earlier we have demonstrated [14], that serum from HUVEC-immunized animals contains antibodies that bind to several HUVEC proteins. Induction of central tolerance achieved by intrathymic injection of HUVEC prior to IP immunization or immunomodulatory therapy with pristane abolished antibody production in the treated rats (Figure 5B). Pre-immune serum did not cross-react with HUVEC proteins. Thus, these findings suggest that the central and peripheral immunomodulatory strategies resulted in a reduction in lung inflammation characterized by a reduced recruitment of 
Table 1 TCR V $\beta$ expression on CD4+ T cells (\%) from lung and spleen of HUVEC and HUVEC-pristane treated rats (n=4)

\begin{tabular}{|c|c|c|c|c|c|c|}
\hline & V $\beta 16.1$ & & $\mathrm{~V} \beta 10$ & & $\mathrm{~V} \beta 8.5$ & \\
\hline Experimental Groups $(n=4)$ & lung & spleen & lung & spleen & lung & spleen \\
\hline Control & $12.1 \pm 0.34$ & $12.7 \pm 0.34$ & $9.7 \pm 0.29$ & $9.2 \pm 0.59$ & $4.2 \pm 0.414$ & $4.4 \pm 0.59$ \\
\hline HUVEC* & $11.5 \pm 0.88$ & $12.1 \pm 0.4$ & $8.22 \pm 0.34$ & $8.08 \pm 0.61$ & $7.1 \pm 1.27$ & $8.4 \pm 2.41$ \\
\hline \multirow[t]{2}{*}{ HUVEC+Pristane* } & $7.67 \pm 0.47$ & $9.05 \pm 0.59$ & $7.1 \pm 0.77$ & $5.62 \pm 1.68$ & $7.79 \pm 1.32$ & $5.7 \pm 1.82$ \\
\hline & ${ }^{*} p \leq 0.008$ & ${ }^{*} p \leq 0.003$ & ${ }^{*} p \geq 0.05$ & ${ }^{*} p \geq 0.05$ & ${ }^{*} p \geq 0.05$ & ${ }^{*} p \geq 0.05$ \\
\hline
\end{tabular}

macrophages, CD4+ T cells and B cells to the rat lung in response to intraperitoneal HUVEC injection.

\section{Discussion}

It has been shown by several groups that alveolar septal cells in the lungs from patients with severe emphysema are undergoing apoptosis [29,33,34], and that induction of apoptosis in mouse lungs indeed causes emphysematous changes [35]. Alveolar septal cell apoptosis is likely the consequence of oxidative stress [36], withdrawal of the impact of lung cell survival factors [28] and/or the action of proteases [37]. We have recently shown that alveolar septal cell apoptosis also occurs in our rat autoimmune emphysema model [14]. Here we apply established immune modulating strategies [19,38,39] to demonstrate that immunity-skewing approaches can modify the autoimmune response to HUVEC immunization, reduce lung cell apoptosis and prevent airspace enlargement.

The thymus plays an important role in acquired tolerance [40], and several studies show that intrathymic injection of alloantigens or autoantigens induces specific systemic tolerance in experimental autoimmune models $[17,41]$. The mechanism by which intrathymic injection of antigen results in systemic tolerance is still elusive, although injection of antigen into the thymus induces apoptosis of thymic $\mathrm{T}$ cells [42]. In addition, an increase in clonotype positive $\mathrm{T}$ cells in the thymus prevents the peripheral expansion of antigen-specific CD4+ T cells [43] and induces prolonged anergy [44].

The $\mathrm{CD}^{+} \mathrm{CD}^{2} 5^{+}$regulatory population of $\mathrm{T}$ cells (Treg cells), which expresses the forkhead family

Table 2 The percentage of Foxp3-expressing CD25+/CD4 $+T$ regulatory cells in rat lungs and spleen. CD4+/CD25 + /Foxp3+

\begin{tabular}{lcc}
\hline Experimental Groups & lung & spleen \\
\hline Control & $0.69 \pm 0.12$ & $0.69 \pm 0.03$ \\
\hline HUVEC & $38.00 \pm 1.60$ & $40.60 \pm 0.52$ \\
\hline Intrathymic-Injection-HUVEC & $56.00 \pm 0.38$ & $52.7 \pm 1.23$ \\
\hline & $p \leq 0.001$ & $p \leq 0.001$ \\
\hline
\end{tabular}

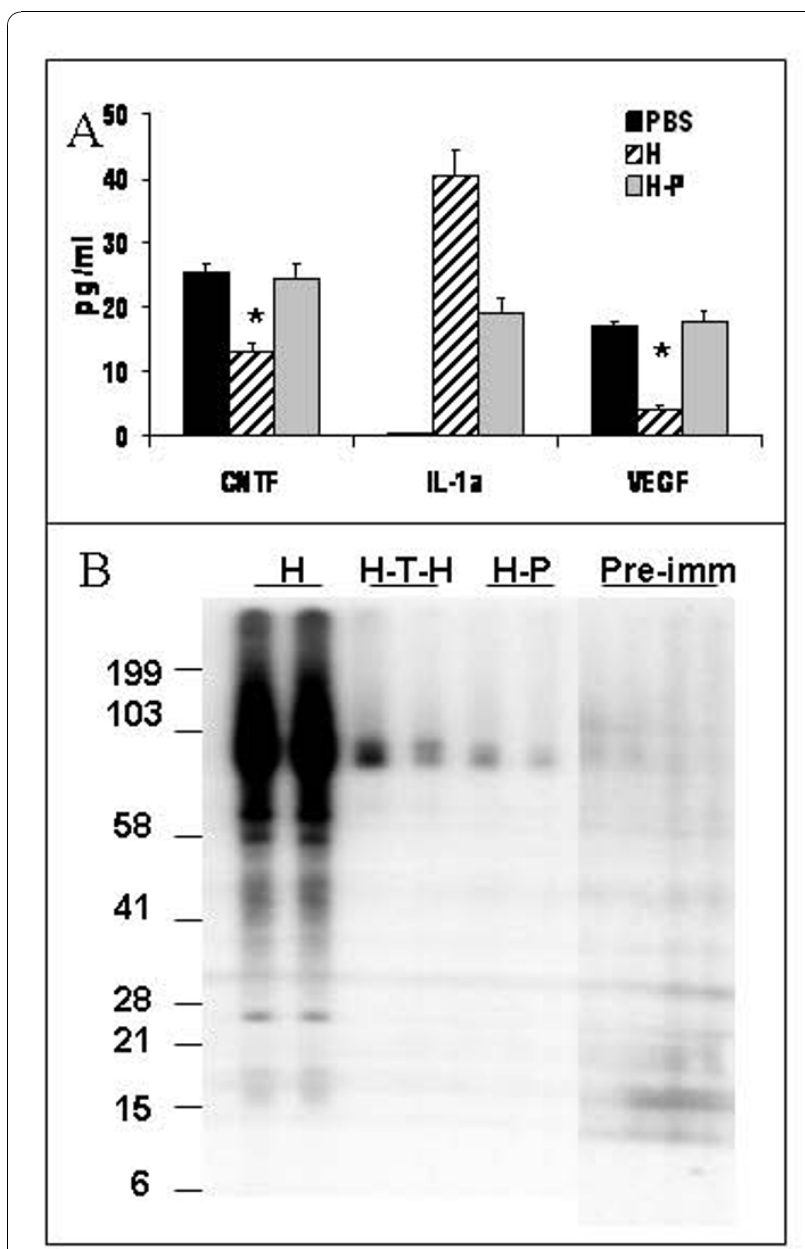

Figure 5 Effects of Immunomodulatory Strategies on Rat Lung Cytokine levels and AECA production. (A) Levels of IL- $\alpha$, VEGF and CNTF in whole lung tissue homogenates from PBS-treated controls, HUVEC-immunized and pristane-pretreated/HUVECimmunized rats measured by Quantibody Rat-1 Array. ${ }^{*} p<0.01$ when compared to the untreated HUVEC-immunized rats. (B) Antibody production in HUVEC-immunized rats. HUVEC protein extracts were separated on $4-12 \%$ gradient Bis-Tris gel and WB was performed using serum (at 1:1000 dilution) from HUVEC-immunized $(\mathrm{H})$ rats (lanes 1 and 2); intrathymically HUVEC-injected and HUVECimmunized (H-T-H) rats (lanes 3 and 4); pristane pre-treated/HUVECimmunized (H-P) animals (lanes 5 and 6); and pre-immune serum (lanes 7-10). 
transcription factor (Foxp3), is the key component of the peripheral tolerance mechanism that protects against a variety of autoimmune diseases [45]. Here we demonstrate that intrathymic injection of HUVEC into 6 week old rats prior to the immunization results in the expansion of CD4+CD25+Foxp3+ Treg cells, suggesting that an expansion of this regulatory $\mathrm{T}$ cell subset may be one of the mechanisms responsible for the protective effect against emphysema development. Whether the mechanism of action for thymic inoculation of HUVEC is attributable to the intrathymic deletion of endothelialreactive $\mathrm{T}$ cells or to the increased production of clonotype-positive $\mathrm{T}$ cells is the subject of ongoing investigations by our group.

In the present experiments, pristane modulated the immune response triggered by HUVEC immunization and prevented AECA production as well as lung cell apoptosis and emphysematous lung destruction. Pristane is considered as an immunological adjuvant namely in vaccines and while administration of the high doses of the hydrocarbon oil adjuvant pristane are used to induce lupus in a murine model of systemic lupus erythematosus [46] it also has been also shown to induce a persistent decrease in phytohemagglutinin responsiveness, consistent with a block of polyclonal $\mathrm{T}$ cell proliferation [18]. As an immune adjuvant, we expected pristane to produce immune skewing. We considered that Toll-like Receptor (TLR) signaling, which is activated by pristane (e.g. requirement of TLR 7 for pristane-induced production of autoantibodies and development of murine lupus nephritis [47]) would be upregulated and therefore immune injury could have been ramped up in the lungs. We did not know at the outset whether pristane would exacerbate autoimmunity or ameliorate it and were surprised about the experimental results.

Similar to human emphysema [48], T cells recruited to the lung in response to HUVEC might express a restricted TCR repertoire, suggesting their recruitment to lung in response to a conventional antigenic stimulus. We queried whether alterations of the TCR repertoire of lung and spleen CD4+ $\mathrm{T}$ cells had occurred as a result of pristane treatment. Our earlier studies did not find significantly expanded TCR-Vbeta subsets in ex vivo human COPD lung samples [48]. However, when cultured in vitro, we found seven major CD4-expressing TCR-Vbeta subset expansions from five of the patients with emphysema. This suggested that severe emphysema is associated with inflammation involving $\mathrm{T}$ lymphocytes that are composed of oligoclonal CD4+ T cells. Here we show that pristane treatment decreased the percentage of CD4+ T cells expressing TCR V $\beta 16.1$ in the lung compared to the expression of $\mathrm{V} \beta 16.1$ on CD4+ T cells from the lungs of HUVEC-immunized rats. It has been reported that treatment with antibody directed against this receptor can facilitate prolonged graft survival [49], suggesting pathogenic nature of rat TCR V $\beta 16.1$. We would like also to point out that while human and mouse TCR nomenclature is well characterized in the Immunogenetics Information System http://www.imgt. org, rat TCR are not in the system making it difficult to compare rat TCR repertoire with known nomenclatures and species.

As with VEGF receptor blockade-induced emphysema [28], HUVEC immunization also leads to a decrease in VEGF protein levels in the lung (Figure 5B). VEGF is likely a critical component of the lung structure maintenance program [50]. Here we demonstrate that pretreatment with pristane abolished antibody production and preserved normal levels of VEGF in HUVEC-immunized animals. Moreover, our data indicate that HUVEC immunization induced the expansion of CD4+ T cells expressing TCR V $\beta 16.1$, which usually are associated with an allergic response [51]. This expansion was significantly blunted upon pre-treatment with pristane, suggesting that this $\mathrm{T}$ cell subset may be the pathogenic $\mathrm{CD} 4+\mathrm{T}$ cell subset in this immune model of emphysema. We also found that the expression of ciliary neurotrophic factor (CNTF) was reduced in HUVECimmunized rat lungs (Figure 5A). CNTF is a cytokine of the interleukin-6 (IL-6) family and its mRNA is widely expressed in the brain, heart, lung, and liver, of the rat, in addition to preferential expression in the sciatic nerve [52]. To our knowledge, this is the first report that CNTF protein expression is reduced in lungs of HUVEC immunized rats, and that immunomodulation with pristane prevents this decrease.

Interleukin- $1 \alpha(\mathrm{IL}-1 \alpha)$ is a $19 \mathrm{kDa}$ proinflammatory cytokine that is a potent mediator of the body's response to inflammation, microbial invasion, tissue injury, and immunological response [53], and has a role in arthritis, Alzheimer's disease and tumor growth [54,55]. In this study, we demonstrated a significant upregulation of IL$1 \alpha$ levels in the HUVEC-immunized rat lung that was prevented by pristane treatment (Figure 5A).

Earlier [14] we have demonstrated that HUVEC injection resulted in the production of antibodies against endothelial cells. Here we show that in the lungs of HUVEC-injected rats, the number of B cells and the expression of cell surface CD19 is increased and that the immunomodulatory strategies attenuated this increase (Figure 3). Recently Sato and colleagues [56] reported that small changes in CD19 expression can induce autoantibody production and suggested that modest changes in the expression or function of regulatory molecules, such as CD19, may shift the balance between tolerance and immunity to autoimmunity [56]. It is possible that downregulation of CD19 in B cells, using immunomodulatory strategies applied by us, also 
reflects a shift toward tolerance in our model of autoimmune emphysema. However, it is also possible that the decrease in CD19 expression could simply reflect decreased numbers of $B$ cells in the lungs each expressing normal levels of CD19. Taken together, the results of our experiments show that rats can be made tolerant to the effects of HUVEC-immunization.

A limitation of our studies is that we have not delineated which specific cell-cell interactions, cytokines, antioxidants or proteases are involved in developing tolerance and whether the different immunomodulatory strategies converge toward a final signaling pathway. However, we can conclude that - as there are multiple factors and conditions which can trigger lung emphysema development $[12,57]$ - there are multiple interventions which can prevent experimental emphysema development.

Emphysema, until recently, has been seen as a problem of proteolysis, not of adaptive immunity. Whereas this is somewhat perplexing because the burning cigarette can be seen as an antigen delivery device, most recently a concept of emphysema pathogenesis based on immune mechanisms is emerging $[11,12]$. Lung gene expression profiling of cigarette smoke-exposed rats demonstrated a sustained increased expression of a number of genes implicated in the innate and adaptive immune responses [58]. Chronic lung cell damage, and in particular apoptosis when combined with ineffective phagocytosis (removal of apoptosed cell bodies) [59], may result in the generation of neoantigen peptides [60] and of autoantibodies. Moreover, tobacco anti-idiotypic antibodies have been identified in serum from smokers [61], and the recent finding of anti-elastin autoantibodies [13] in patients with tobacco smoking-induced emphysema, that correlated with severity of the disease, link emphysema to adaptive immunity against a specific lung antigen and suggest the potential for autoimmune pathology.

Whether "priming" strategies can also protect against other - non-HUVEC-induced forms of emphysema remains to be investigated. We are intrigued that experimental manipulations as diverse as intrathymic antigen injection and intraperitoneal delivery of pristane prevented the accumulation of lung $B$ cells and macrophages, facilitated the expansion of CD4+CD25+Foxp3+ Treg cells and suppressed the expansion of TCR V $\beta 16.1$ expressing $\mathrm{T}$ cells in the HUVEC-immunized animals. Whether or not TCR V $\beta 16.1$ expressing $\mathrm{T}$ cells are associated with autoimmune emphysema still need to be verified. Whether or not this is true in human disease needs to be determined. Also, the role of macrophages in this autoimmune emphysema model deserves further investigation.

\section{Conclusions}

The experimental autoimmune emphysema may evolve from a confluence of subtle multigenic variations in cell surface signaling molecules. As in NOD diabetes, where interventions prior to the development of diabetes skew immune responses and are associated with decreased inflammation around pancreatic islets and prevent diabetes $[62,63]$, here we demonstrate that similar immunomodulatory strategies that suppress autoreactive $T$ and $B$ cells and upregulate Treg cells can prevent experimental autoimmune emphysema.

\section{List of Abbreviations}

(AECA): Anti-endothelial cell antibodies; (HUVEC): human umbilical vein endothelial cells; (NOD): non-obese diabetic; (MLI): mean linear intercept; (TUNEL): Terminal deoxynucleotidyl transferase-mediated dUTP nick endlabeling; (TCR): T cell Receptor; (Treg): T regulatory cells; (IHC): immunohistochemistry; (VEGF): vascular endothelial growth factor; (CNTF): neurotrophic factor; (IL-1 $\alpha)$ : interleukin 1alpha; (CINC-2 also known as CXCL3/GROgamma/DCIP-1): cytokine induced neutrophil chemoattractant-2; (CINC-3 also known as CXCL3/GRObeta/MIP-2): cytokine induced neutrophil chemoattractant-3; (GM-CSF): granulocyte-Macrophage Colony Stimulating Factor; (IL-4): interleukin-4; (LIX/CXCL5): lipopolysaccharide-induced CXC chemokine; $(\beta-N G F)$ : nerve growth factor beta; (CX3CL1): Fractalkine.

\section{Acknowledgements}

We gratefully acknowledge Ji-Hyun Lee and Liudas Slepikas for technical assistance with TUNEL and T cell staining, and Dr. John Stewart for critical reading of the manuscript. This study was supported by NIH grant HL60195, Colorado Tobacco Research Grant 31-013, FAMRI grant 072053, AHA grant 0735388, the Hart Family Emphysema Chair and the Bixler COPD Foundation.

\section{Author details}

${ }^{1}$ Department of Medicine, University of Colorado Denver, Aurora, CO, 80045, USA. ${ }^{2}$ VA Palo Alto Health Care System, Stanford University, Palo Alto, CA 94304, USA. ${ }^{3}$ Pulmonary and Critical Care Medicine Division and Virginia Johnson Center for Emphysema Research, Virginia Commonwealth University, Richmond, VA, 23298, USA.

\section{Authors' contributions}

$\mathrm{MH}$ conducted the experiments, drafted the manuscript and performed statistical analysis; MRN participated in study design; APF participated in study design; DK participated in the animal experiments; DGM performed flow cytometry and data analysis; AK and JS have been involved in work leading to the revised the manuscript; VK participated in data acquisition; NB carried out western blots; NFV participated in the design of the study and helped to draft the manuscript and LT-S. conceived of the study and participated in its design and coordination and helped to draft the manuscript. All authors read and approved the final manuscript.

\section{Competing interests}

The authors declare that they have no competing interests.

Received: 21 June 2010 Accepted: 16 December 2010

Published: 16 December 2010

\section{References}

1. Agusti AG, Noguera A, Sauleda J, Sala E, Pons J, Busquets X: Systemic effects of chronic obstructive pulmonary disease. Eur Respir J 2003, 21:347-360.

2. Wouters EF: Local and systemic inflammation in chronic obstructive pulmonary disease. Proc Am Thorac Soc 2005, 2:26-33.

3. de Jong JW, Belt-Gritter B, Koeter GH, Postma DS: Peripheral blood lymphocyte cell subsets in subjects with chronic obstructive pulmonary 
disease: association with smoking, lgE and lung function. Respir Med 1997, 91:67-76.

4. Noguera A, Busquets X, Sauleda J, Villaverde JM, MacNee W, Agusti AG: Expression of adhesion molecules and $G$ proteins in circulating neutrophils in chronic obstructive pulmonary disease. Am J Respir Crit Care Med 1998, 158:1664-1668.

5. Schols AM, Buurman WA, Staal van den Brekel AJ, Dentener MA, Wouters EF: Evidence for a relation between metabolic derangements and increased levels of inflammatory mediators in a subgroup of patients with chronic obstructive pulmonary disease. Thorax 1996, 51:819-824.

6. Cosio MG, Guerassimov A: Chronic obstructive pulmonary disease. Inflammation of small airways and lung parenchyma. Am J Respir Crit Care Med 1999, 160:S21-S25.

7. Saetta M, Turato G, Maestrelli P, Mapp CE, Fabbri LM: Cellular and structural bases of chronic obstructive pulmonary disease. Am J Respir Crit Care Med 2001, 163:1304-1309.

8. Hogg JC, Chu F, Utokaparch S, Woods R, Elliott WM, Buzatu L, et al: The nature of small-airway obstruction in chronic obstructive pulmonary disease. N Engl J Med 2004, 350:2645-2653.

9. Cosio MG, Majo J, Cosio MG: Inflammation of the airways and lung parenchyma in COPD: role of T cells. Chest 2002, 121:160S-165S.

10. Hodge $S$, Hodge G, Holmes M, Reynolds PN: Increased airway epithelial and T-cell apoptosis in COPD remains despite smoking cessation. Eur Respir J 2005, 25:447-454

11. Agusti A, MacNee W, Donaldson K, Cosio M: Hypothesis: does COPD have an autoimmune component? Thorax 2003, 58:832-834

12. Cosio MG, Saetta M, Agusti A: Immunologic aspects of chronic obstructive pulmonary disease. N Engl J Med 2009, 360:2445-2454.

13. Lee SH, Goswami S, Grudo A, Song LZ, Bandi V, Goodnight-White S, et al: Antielastin autoimmunity in tobacco smoking-induced emphysema. Nat Med 2007, 13:567-569.

14. Taraseviciene-Stewart L, Scerbavicius R, Choe KH, Moore M, Sullivan A, Nicolls MR, et al: An animal model of autoimmune emphysema. Am J Respir Crit Care Med 2005, 171:734-742.

15. Chatenoud L, Thervet E, Primo J, Bach JF: Anti-CD3 antibody induces longterm remission of overt autoimmunity in nonobese diabetic mice. Proc Natl Acad Sci USA 1994, 91:123-127.

16. Pasquali L, Giannoukakis N, Trucco M: Induction of immune tolerance to facilitate beta cell regeneration in type 1 diabetes. Advanced Drug Delivery Reviews 2008, 60:106-113.

17. Ilan Y, Attavar P, Takahashi M, Davidson A, Horwitz MS, Guida J, et al: Induction of central tolerance by intrathymic inoculation of adenoviral antigens into the host thymus permits long-term gene therapy in Gunn rats. J Clin Invest 1996, 98:2640-2647.

18. Freund YR, Blair PB: Depression of natural killer activity and mitogen responsiveness in mice treated with pristane. J Immunol 1982. 129:2826-2830.

19. Sprent J, Kishimoto $\mathrm{H}$ : The thymus and central tolerance. Transplantation 2001, 72:S25-S28.

20. Naji A: Induction of tolerance by intrathymic inoculation of alloantigen. Curr Opin Immunol 1996, 8:704-709.

21. Fukada J, Kurimoto Y, Ruiz P, Aitouche A, Zeevi A, Li S, et al: Long-term survival of rat cardiac allografts by intrathymic plus portal venous injections of donor bone marrow cells and short-term tacrolimus immunosuppression. Transpl Int 2001, 14:311-319.

22. Cober SR, Randolph MA, Lee WP: Skin allograft survival following intrathymic injection of donor bone marrow. J Surg Res 1999, 85:204-208.

23. Li S, Louis LB, Kawaharada N, Yousem SA, Pham SM: Intrathymic inoculation of donor bone marrow induces long-term acceptance of lung allografts. Ann Thorac Surg 2003, 75:257-263.

24. Bruneel A, Labas V, Mailloux A, Sharma S, Vinh J, Vaubourdolle M, et al: Proteomic study of human umbilical vein endothelial cells in culture. Proteomics 2003, 3:714-723.

25. Chu Q, Moreland RJ, Gao L, Taylor KM, Meyers E, Cheng SH, Scheule RK: Induction of Immune Tolerance to a Therapeutic Protein by Intrathymic Gene Delivery. Mol Ther 2010, 18:2146-2154.

26. Marodon $G$, Klatzmann D: In situ transduction of stromal cells and thymocytes upon intrathymic injection of lentiviral vectors. BMC Immunol 2004, 5:18.
27. Marodon G, Fisson S, Levacher B, Fabre M, Salomon BL, Klatzmann D: Induction of antigen-specific tolerance by intrathymic injection of lentiviral vectors. Blood 2006, 108:2972-2978.

28. Kasahara Y, Tuder RM, Taraseviciene-Stewart L, Le Cras TD, Abman S, Hirth PK, et al: Inhibition of VEGF receptors causes lung cell apoptosis and emphysema. J Clin Invest 2000, 106:1311-1319.

29. Imai K, Mercer BA, Schulman LL, Sonett JR, D'Armiento JM: Correlation of lung surface area to apoptosis and proliferation in human emphysema. Eur Respir J 2005, 25:250-258.

30. Finotto S, Eigenbrod T, Karwot R, Boross I, Doganci A, Ito H, et al: Local blockade of IL-6R signaling induces lung CD4+ $\mathrm{T}$ cell apoptosis in a murine model of asthma via regulatory T cells. Int Immunol 2007, 19:685-693.

31. Mack DG, Lanham AK, Palmer BE, Maier LA, Watts TH, Fontenot AP: 4-1BB enhances proliferation of beryllium-specific $T$ cells in the lung of subjects with chronic beryllium disease. J Immunol 2008, 181:4381-4388,

32. Zozulya AL, Wiendl H: The role of regulatory T cells in multiple sclerosis. Nat Clin Pract Neurol 2008, 4:384-398.

33. Kasahara Y, Tuder RM, Cool CD, Lynch DA, Flores SC, Voelkel NF: Endothelial cell death and decreased expression of vascular endothelial growth factor and vascular endothelial growth factor receptor 2 in emphysema. Am J Respir Crit Care Med 2001, 163:737-744.

34. Yokohori N, Aoshiba K, Nagai A: Increased levels of cell death and proliferation in alveolar wall cells in patients with pulmonary emphysema. Chest 2004, 125:626-632.

35. Aoshiba K, Yokohori N, Nagai A: Alveolar wall apoptosis causes lung destruction and emphysematous changes. Am J Respir Cell Mol Biol 2003, 28:555-562.

36. MacNee W: Oxidative stress and lung inflammation in airways disease. Eur J Pharmacol 2001, 429:195-207.

37. Shapiro SD: Proteinases in chronic obstructive pulmonary disease. Biochem Soc Trans 2002, 30:98-102.

38. Chan C, Lechler Rl, George AJ: Tolerance mechanisms and recent progress. Transplant Proc 2004, 36:561S-569S.

39. Goldschneider I, Cone RE: A central role for peripheral dendritic cells in the induction of acquired thymic tolerance. Trends Immunol 2003, 24:77-81.

40. Kappler JW, Roehm N, Marrack P: T cell tolerance by clonal elimination in the thymus. Cell 1987, 49:273-280.

41. Posselt AM, Barker CF, Friedman AL, Naji A: Prevention of autoimmune diabetes in the $B B$ rat by intrathymic islet transplantation at birth. Science 1992, 256:1321-1324.

42. Chen W, Sayegh MH, Khoury SJ: Mechanisms of acquired thymic tolerance in vivo: intrathymic injection of antigen induces apoptosis of thymocytes and peripheral T cell anergy. J Immunol 1998, 160:1504-1508.

43. Chen W, Issazadeh S, Sayegh MH, Khoury SJ: In vivo mechanisms of acquired thymic tolerance. Cell Immunol 1997, 179:165-173.

44. Khoury SJ, Gallon L, Chen W, Betres K, Russell ME, Hancock WW, et al: Mechanisms of acquired thymic tolerance in experimental autoimmune encephalomyelitis: thymic dendritic-enriched cells induce specific peripheral T cell unresponsiveness in vivo. J Exp Med 1995, 182:357-366.

45. Pacholczyk R, Kern J: The T-cell receptor repertoire of regulatory $T$ cells. Immunology 2008, 125:450-458.

46. Reeves WH, Lee PY, Weinstein JS, Satoh M, Lu L: Induction of autoimmunity by pristane and other naturally occurring hydrocarbons. Trends Immunol 2009, 30:455-464.

47. Savarese E, Steinberg C, Pawar RD, Reindl W, Akira S, Anders HJ, et al: Requirement of Toll-like receptor 7 for pristane-induced production of autoantibodies and development of murine lupus nephritis. Arthritis Rheum 2008, 58:1107-1115.

48. Sullivan AK, Simonian PL, Falta MT, Mitchell JD, Cosgrove GP, Brown KK, et al: Oligoclonal $C D 4+T$ cells in the lungs of patients with severe emphysema. Am J Respir Crit Care Med 2005, 172:590-596.

49. Aspinall R, Kampinga J: A novel lymphocyte surface antigen recognized by the monoclonal antibody HIS45. Thymus 1989, 13:245-252.

50. Voelkel NF, Vandivier RW, Tuder RM: Vascular endothelial growth factor in the lung. Am J Physiol Lung Cell Mol Physiol 2006, 290:L209-L221.

51. Beyer K, Hausler T, Kircher M, Nickel R, Wahn U, Renz H: Specific V beta T cell subsets are associated with cat and birch pollen allergy in humans. J Immunol 1999, 162:1186-1191. 
52. Ohta $K$, Hara H, Hayashi $K$, Itoh N, Ohi T, Ohta M: Tissue expression of rat ciliary neurotrophic factor (CNTF) mRNA and production of the recombinant CNTF. Biochem Mol Biol Int 1995, 35:283-290.

53. Dinarello CA: Interleukin-1, interleukin-1 receptors and interleukin-1 receptor antagonist. Int Rev Immunol 1998, 16:457-499.

54. Infante J, Llorca J, Mateo I, Rodriguez-Rodriguez E, Sanchez-Quintana C, Sanchez-Juan $P$, et al: Interaction between poly(ADP-ribose) polymerase 1 and interleukin $1 \mathrm{~A}$ genes is associated with Alzheimer's disease risk. Dement Geriatr Cogn Disord 2007, 23:215-218.

55. Havemose-Poulsen A, Sorensen LK, Bendtzen K, Holmstrup P: Polymorphisms within the IL-1 gene cluster: effects on cytokine profiles in peripheral blood and whole blood cell cultures of patients with aggressive periodontitis, juvenile idiopathic arthritis, and rheumatoid arthritis. J Periodontol 2007, 78:475-492.

56. Sato S, Hasegawa M, Fujimoto M, Tedder TF, Takehara K: Quantitative genetic variation in CD19 expression correlates with autoimmunity. $J$ Immunol 2000, 165:6635-6643.

57. Taraseviciene-Stewart L, Voelkel NF: Molecular pathogenesis of emphysema. J Clin Invest 2008, 118:394-402.

58. Gebel S, Gerstmayer B, Kuhl P, Borlak J, Meurrens K, Muller T: The kinetics of transcriptomic changes induced by cigarette smoke in rat lungs reveals a specific program of defense, inflammation, and circadian clock gene expression. Toxicol Sci 2006, 93:422-431.

59. Vandivier RW, Henson PM, Douglas IS: Burying the dead: the impact of failed apoptotic cell removal (efferocytosis) on chronic inflammatory lung disease. Chest 2006, 129:1673-1682.

60. Mahoney JA, Rosen A: Apoptosis and autoimmunity. Curr Opin Immunol 2005, 17:583-588.

61. Koethe SM, Kuhnmuench JR, Becker CG: Neutrophil priming by cigarette smoke condensate and a tobacco anti-idiotypic antibody. Am J Pathol 2000, 157:1735-1743.

62. Cooke A, Phillips JM, Parish NM: Tolerogenic strategies to halt or prevent type I diabetes. Nature Immunology 2001, 2:810-815.

63. Rosmalen JGM, van Ewijk V, Leenen PJM: T-cell education in autoimmune diabetes: teachers and students. Trends in Immunology 2002, 23:40-46.

doi:10.1186/1465-9921-11-179

Cite this article as: Hanaoka et al:: Immunomodulatory strategies prevent the development of autoimmune emphysema. Respiratory Research 2010 11:179.

\section{Submit your next manuscript to BioMed Central and take full advantage of:}

- Convenient online submission

- Thorough peer review

- No space constraints or color figure charges

- Immediate publication on acceptance

- Inclusion in PubMed, CAS, Scopus and Google Scholar

- Research which is freely available for redistribution 